\title{
UNA PROFESIÓN RECONOCIDA EN CONTEXTOS DE SALUD: LA EDUCACIÓN ARTÍSTICA $^{1}$
}

\section{An acknoledged profession in health context: Artistic education}

\author{
Noemí Ávila \\ Universidad Complutense de Madrid \\ noemi.avila@art.ucm.es \\ María ACASO \\ Universidad Complutense de Madrid \\ macaso@art.ucm.es
}

Recibido: 3 de febrero de 2011

Aprobado: 6 de mayo de 2011

\section{Resumen:}

El arte y la creatividad son conceptos que están comenzando a vincularse en los ámbitos sanitarios españoles. La clave para que puedan desarrollarse con todas sus potencialidades reside en la profesionalización de la educación artística. Los educadores artísticos utilizan el arte y la creación como mediadores en espacios de salud. Solo el reconocimiento y posicionamiento de estos profesionales en el organigrama de la salud en España pueden abrir posibilidades para la mejora de la calidad de vida de los pacientes. Palabras clave: educación artística, arte y salud, cuidados de salud, calidad de vida.

Ávila, N., Acaso, M. 2011: Una profesión reconocida en contextos de salud: la educación artística. Arte, Individuo y Sociedad, Vol. 23, Núm. Especial, 19-27.

\begin{abstract}
:
Art and creativity are subjects which are begining to introduce in health enviroments in Spain. The key in order to offer more possibilities about this relationship is that we recognize the art educator in this context. Art educator uses art and creation like a tool of intervention in this kind of enviroments. It is necesary recognize and this job in order to improve the healthcare of patients, from another point of view.
\end{abstract}

Key words: art education, art \& health, healthcare.

Sumario: 1. Del voluntario al EDUCADOR en contextos de Salud. 2. La realidad de la educación artística hoy en contextos sanitarios. 3. Hacia qué modelo queremos ir con la especialización de profesionales en el área. 4. Herramientas para la educación artística en contextos de salud. Referencias.

\footnotetext{
${ }^{1}$ Esta investigación ha sido financiada por el Ministerio de Ciencia e Innovación (EDU200805441-C02-00/EDU)
} 


\section{Del voluntario al EDUCADOR en contextos de salud}

Trabajar enseñando arte, ser profesor o docente en el ámbito de las artes plásticas es una profesión reconocida, pero este reconocimiento se suele diluir cuando el contexto en el que se trabaja no se considera un contexto legítimo. Reconocemos a un docente de arte en la escuela, en un instituto, en la universidad, en un museo e incluso en un centro cultural. Pero, ¿reconocemos su labor cuando realiza esta tarea en un hospital?, ¿o cuando trabaja en un centro de mayores? ¿¿cómo llamamos a este profesional que dedica horas realizando talleres de arte y creatividad con pacientes y ancianos? La realidad es que muy pocas veces reconocemos a este profesional y seguramente lo confundamos con un voluntario, un trabajador social o un terapeuta.

Por otro lado, la sociedad demanda que en hospitales, centros de mayores, residencias, centros de acogida, etc., existan programas alternativos, programas de educación o programas de ocio y tiempo libre que mejoren las condiciones y la calidad de vida de los pacientes hospitalizados, ancianos o adolescentes.

La cuestión es, si se reconoce la necesidad de estos programas y actividades alternativas, ¿por qué no se reconoce al profesional que las realiza?

Reconocer la labor de un profesional es dar el paso del voluntario al profesional. Y aunque en la teoría la labor del voluntario nunca debe sustituir la labor de un profesional, en la práctica, las dinámicas que se están llevando a cabo en estos contextos de salud son bien distintas. Las acciones institucionales no cubren la demanda de este tipo de programas, y son las asociaciones de familiares, enfermos o pacientes, o bien fundaciones las que generan este tipo de actividades, en la mayor parte de los casos con poca especialización en la materia, y apoyados por el trabajo de los voluntarios, $\mathrm{y}$ en muy contadas ocasiones con apoyo financiero.

En cualquier caso estas dinámicas apenas tienen difusión ni continuidad, con lo cual, implementar y asentar estas propuestas artísticas y creativas en estos ámbitos resulta una tarea compleja.

Observando las dinámicas que se están produciendo en otros países (Inglaterra, Canadá, Australia), el arte incorporado en contextos de Salud ha pasado por varias fases, siendo la "profesionalización" y "especialización" del educador artístico una de las primeras fases en estos países de referencia en el ámbito de Arte y Salud. Cuando se incorpora a este profesional en el engranaje de cualquier sistema sanitario puede desarrollar su labor y comenzar también los procesos de intercambio y desarrollo conjunto con otros profesionales del ámbito sanitario (médicos, enfermeros, etc.).

En cualquier caso, es necesario revisar este proceso de incorporación y reconocimiento del educador artístico en Salud, y establecer las siguientes fases:

FASE 1: Incorporación y reconocimiento del Arte, la Creatividad y la Educación artística como recurso de bienestar en contextos de Salud.

FASE 2: Formación de un profesional especializado. En la actualidad, No existe un profesional en Arte, Creatividad y Educación en contextos de Salud, reconocido en España, porque no existe tampoco una formación específica en el tema, la Universidad Complutense de Madrid tiene un Curso de Experto en el área y hay previstos un máster y un doctorado próximamente. 
FASE 3: Asimilación del profesional en Arte y Salud dentro de los contextos sanitarios.

\section{La realidad de la educación artística hoy en contextos sanitarios}

Como ya se ha explicado en las fases del posicionamiento del educador artístico en contextos de salud, es importante reflexionar sobre las necesidades dentro de la estructura sanitaria, atender a los espacios y tiempos del paciente y definir espacio y tiempo de acción.

Un ejemplo de esta gestión de espacios y tiempos para el arte y la creatividad (Ullán, A. Y Belver, M.H., 2004) podría ser el diagrama que utilizamos para entender los procesos de hospitalización de niños y adolescentes y como en ese triángulo donde se interrelacionan el tiempo para el diagnostico, tratamiento y cuidado físico, el tiempo educativo y el tiempo libre.

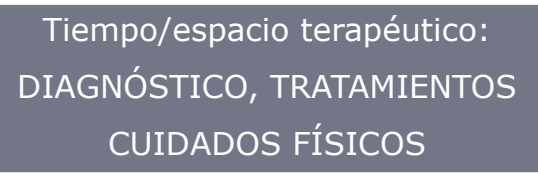

Espacios en la hospitalización infantil-adolescente

Tiempo en la hospitalización infantil-adolescente

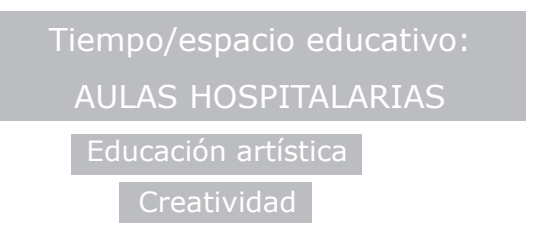

Tiempo/espacio educativo: AULAS HOSPITALARIAS Creatividad

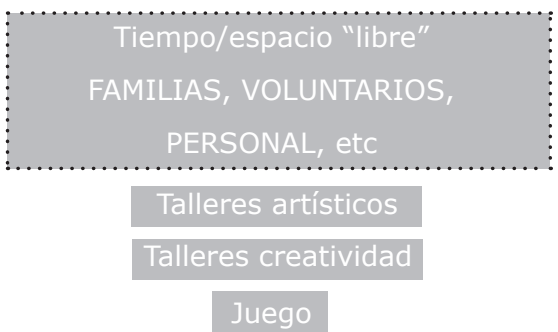

Fig. 1. El Arte, la educación artística y la creatividad en el diagrama de tiempos y espacios en la hospitalización infantil y adolescente.

Con respecto a los niños y adolescentes hay una especial sensibilización que ha facilitado el espacio y el tiempo a los educadores. Pero esta labor de reconocimiento no es tan sencilla cuando pasamos al mundo del adulto.

$\mathrm{Si}$ atendemos a la siguiente figura podemos ver que existen dos capas concéntricas, en torno al núcleo de tratamientos terapéuticos y médicos. Estas dos capas concéntricas son las que nos hablan de la calidad de vida del paciente, y como factor de medición de esa calidad de vida del paciente, tenemos una capa bien definida, formada por los terapeutas, psicólogos, y maestros etc., cuya labor y reconocimiento está 
perfectamente legitimada. Pero estos profesionales no pueden cubrir todas la necesidades y por encima de esta capa podemos ver otras más diluidas que la conforman los voluntarios, familiares, monitores, etc. Uno de los objetivos de la implementación de talleres artisticos en espacios sanitarios es pasar a formar parte de la primera capa concéntrica, reconociendo la labor de la educación artística en el ámbito de la salud.

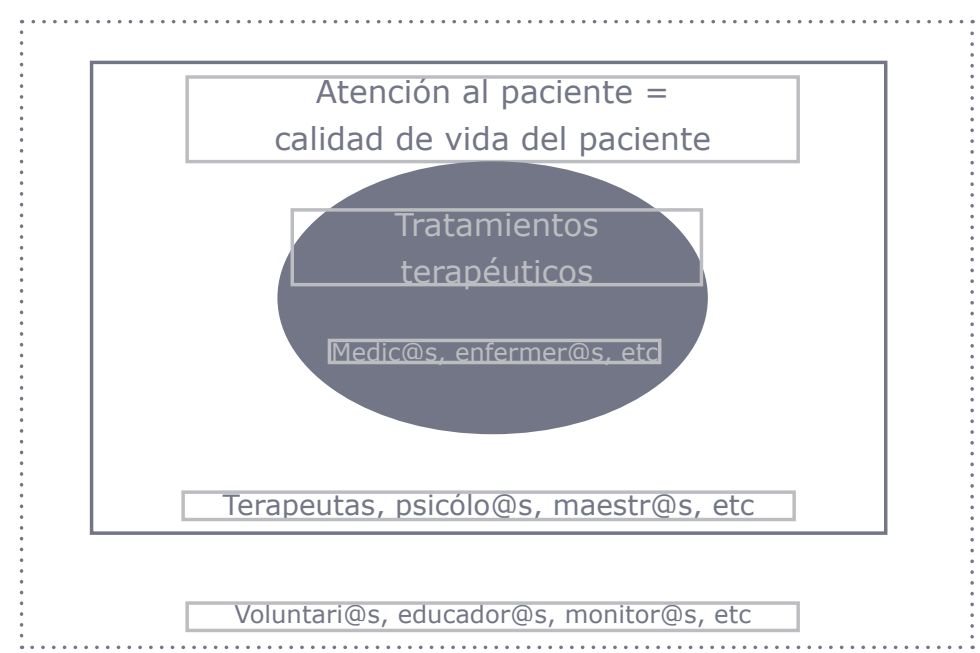

Fig. 2. Diagrama del posicionamiento del Educador Artístico en Contextos de Salud.

\section{Hacia qué modelos queremos ir con la especialización de profesionales en el área}

El desarrollo del área del Arte y la Salud en España es muy reciente. Las referencias más interesantes hay que buscarlas en Inglaterra, donde este ámbito tiene ya cierta tradición, con acciones, profesionales e investigaciones con el suficiente rigor como para ser revisadas y analizadas para poder ser implementadas en nuestra realidad sanitaria.

En España, aunque aún de forma puntual existen propuestas que empiezan a incorporar especialistas en Arte y Salud. En el Hospital provincial de Castellón, el artista Josep María Martín ha generado el "Prototipo de espacio para gestionar las emociones en el hospital", una instalación artística integrada en el espacio hospitalario. Otro ejemplo interesante es el Hospital de Denia, que ha sido concebido desde su inicio con un proyecto de arte vinculado (cuidArt) que incorpora espacios expositivos, talleres y dinámicas artísticas, y una directora de arte.

Desde los museos y centros de arte también se han desarrollado algunas propuestas, Artium, por ejemplo, trabaja con los pacientes del Hospital Psiquiátrico de Alava desde el año 2004, y recientemente con el Hospital de día de Adicciones.

En cualquier caso, se trata de acciones puntuales, y es necesario dibujar el mapa de las posibles relaciones y dinámicas que puedan establecer el Arte y la Salud en nuestro contexto sanitario. Uno de los esquemas de referencia para poder comprender el ámbito de acción que abarcaría todo lo relacionado con el Arte y la Salud, es el diagrama que propone Mc Naugthon, en el que se interrelacionan los conceptos arte y salud, y por otro lado, las acciones, los agentes y colectivos implicados: 




PROYECTOS ARTÍSTICOS SOCIALES

Proyectos de arte y creación para

mejorar las relaciones sociales.

Estudios que reflejan que las salud.
SOCIAL

\section{COMUNIDADES}

Proyectos orientados a generar comunidades que usen métodos creativos para explorar, difundir y comunicar mensajes relacionados con la salud y el bienestar.
ARTE, CREATIVIDAD Y BIENESTAR

Proyectos que enfatizan la

importancia de la creatividad en el bienestar. El objetivo es

trabajar con personas para qué comprendan su salud.

Fig. 3. El Diamante del Arte y la Salud (traducción) (McNaughton et al. 2005)

Lo que propone este diagrama es la interrelación entre los servicios de salud y el arte a través de dinámicas que incorporen nuevos profesionales al ámbito sanitario (educadores, artistas, curadores, creativos) y que generen nuevas dinámicas sociales que desarrollen propuestas y programas de mejora de calidad de vida con respecto a la salud.

Este modelo que integra arte y salud, se reconoce en otros países como Arts\&Health, un campo de acción, estudio e investigación que reflexiona sobre como las artes pueden contribuir a la salud, el bienestar, la inclusión social y la calidad de vida de las personas.

Desde el año 2003, la Universidad Complutense de Madrid y la Universidad de Salamanca (Departamento de Didáctica de la Expresión Plástica de la Universidad Complutense de Madrid, el Museo Pedagógico de Arte Infantil y Departamento de Psicología Social de la Universidad de Salamanca) están desarrollando una serie de investigaciones en torno a las posibilidades de mejora de los niños y adolescentes hospitalizados a través del juego, la creatividad y el arte, enmarcadas en el proyecto curarte $\mathrm{I}+\mathrm{D}$.

El proyecto curarte I+D surge como una propuesta multidisciplinar y pluriinstitucional, para avanzar en la mejora de las condiciones de vida de los niños y adolescentes hospitalizados, desarrollando actividades de juego y creativas especialmente concebidas para responder a sus necesidades específicas y promover estas actividades como recurso de bienestar y salud.

En la actualidad el proyecto curarte I+D ha ampliado su campo de acción, con propuestas para adultos y Tercera Edad. Los objetivos del mismo se han ampliado, y en estos momentos trabajamos en el desarrollo de programas de diseño, implementación y evaluación de arte, creación y educación artística en contextos de Salud.

Las investigaciones del proyecto curarte $\mathrm{I}+\mathrm{D}$ tienen como objetivo generar un campo de conocimiento y estudio en el área del Arte y la Salud en España. 
Desde el proyecto curarte I+D, continuando con el esquema propuesto por $\mathrm{McN}$ aughton, se han desarrollado varias acciones que ilustran las relaciones que el Arte y la Salud potencialmente pueden establecer:



ARTE, CREATIVIDAD Y BIENESTAR

- Programas de Talleres con niños y adolescentes hospitalizados

- Programas de Talleres con enfermos de Alzheimer $y$ otras demencias

INDIVIDUAL

APOYO A SERVICIOS DE SALUD -Proyectos de "humanización" de espacios pediátricos

-Proyecto "Efectos del juego simbólico sobre el alivio del dolor en niños hospitalizados". Hospital Universitario de Salamanca

Fig. 3. Acciones proyecto curarte I $+\mathrm{D}$, siguiendo la estructura del Diamante del Arte y la Salud propuesta por McNaughton et al. (2005)

Los investigadores del proyecto curarte $\mathrm{I}+\mathrm{D}$, a partir de estas acciones, están generando un campo de acción y de conocimiento para convertir en realidades justificadas y necesarias en los espacios sanitarios, dinámicas como programas de arte y de creación para pacientes, familiares o personal sanitario, humanización de espacios pediátricos, etc.

Continuando en la línea de especializar al profesional en el área del Arte y la Salud, nos encontramos de nuevo con la dificultad para establecer unas directrices o metodologías comunes. Existen múltiples propuestas, dispares y poco documentadas. La mayor parte de ellas llevadas a cabo por voluntariado de asociaciones, colectivos o familiares de enfermos y familiares que trabajan con la plástica y la creatividad como instrumento en talleres con diferentes objetivos (educativos, ocio, salud, terapia, etc.) . Pero esta abundancia y falta de criterios comunes no debe impedir generar un estudio riguroso y plantear unas directrices para realizar un trabajo profesional.

Otros países (como EEUU o Australia) hacen especial hincapié en que el punto de partida para generar este nuevo campo de estudio y de investigación y en definitiva de profesionalización, es la necesidad de crear bases de datos que permitan ordenar y sistematizar las acciones (casi todas ella dependientes de movimientos sociales, comunitarios o culturales) y a partir de esta sistematización poder generar una campo para la evaluación y la investigación. 
En Inglaterra, uno de los países con un mayor desarrollo en este ámbito, se describen las siguientes dificultades en los procesos de evaluación e investigación cuando se vinculan arte y salud (Clift et al, 2009):

- La complejidad y la sutilidad de la propia realidad del Arte y la Creación

- El amplio espectro de propuestas y formas artísticas

- La diversidad de los agentes que participan en los proyectos de Arte y Salud

- La gran variedad de temas de salud que pueden ser tratados

Así pues, no es tan sencillo hablar de un rigor metodológico en las prácticas que introducen el arte y la creatividad en contextos sanitarios, precisamente por las razones anteriormente mencionadas y especialmente por la complejidad y la diversidad de cada uno de los contextos potenciales de acción.

En el siguiente apartado, planteamos un punto de partida para establecer precisamente un cierto rigor metodológico en las acciones que introducen el arte a través talleres en espacios sanitarios.

\section{Herramientas para la educación artística en contextos de salud}

Las investigaciones vinculadas al proyecto curarte $\mathrm{I}+\mathrm{D}$, plantean que la mejora de los contextos de Salud, desde la perspectiva de sus investigaciones y la labor de sus educadores, se inicia en el momento en que el paciente pueda percibir experiencias positivas, que favorezcan sus posibilidades de normalización, y en esto el arte tiene mucho que aportar.

La tarea del educador se centra en modificar la percepción del contexto de Salud, reformulando dispositivos existentes y/o introduciendo nuevos dispositivos, para generar experiencias positivas y enriquecedoras desde el arte y la creación.

Teniendo clara estas premisas, podemos empezar a trabajar aplicando unas metodologías didácticas siempre ajustadas a la realidad del paciente o del participante en el taller.

La experiencia en los últimos años, nos lleva indicar la necesidad de trabajar en un continuo feedback que nos permita ajustar tanto metodologías didácticas como las metodologías de evaluación al contexto de salud en concreto en el que estamos, por eso nos referimos a talleres ajustados (Pascale, P. y Avila, N., 2007).

En el diseño, implementación y evaluación de estos talleres ajustados en contextos de Salud se deberían tener en cuenta al menos los siguientes aspectos básicos:

1. Elección del tipo de taller a realizar en función de sus objetivos prioritarios:

Artísticos (definido por dinámicas artísticas)

Educativos (definido por fines educativos)

De Salud (definido por objetivos de Salud)

Híbridos (Compatibilidad/Incompatibilidad de objetivos) 
2. Metodologías ajustadas para los objetivos prioritarios definidos (Compatibilidad/Incompatibilidad de metodologías)

3. Evaluación de los talleres en función de los objetivos definidos (Compatibilidad/Incompatibilidad de las evaluaciones)

En cada uno de estos tres aspectos contemplados, se hace referencia a las compatibilidades e incompatibilidades, en realidad nos estamos refiriendo de nuevo al ajuste al contexto. En un determinado taller un objetivo prioritario de carácter artístico (como pueda ser el conocimiento de artistas contemporáneos que trabajan con el cuerpo) puede entrar en conflicto con el contenido específico de un taller de salud en un contexto con adolescentes con trastornos alimenticios. Por eso hacemos tanto hincapié es establecer los objetivos prioritarios de cada taller y ajustar tanto los contenidos, metodologías y evaluaciones para que no se produzcan conflictos ni incompatibilidades de este tipo.

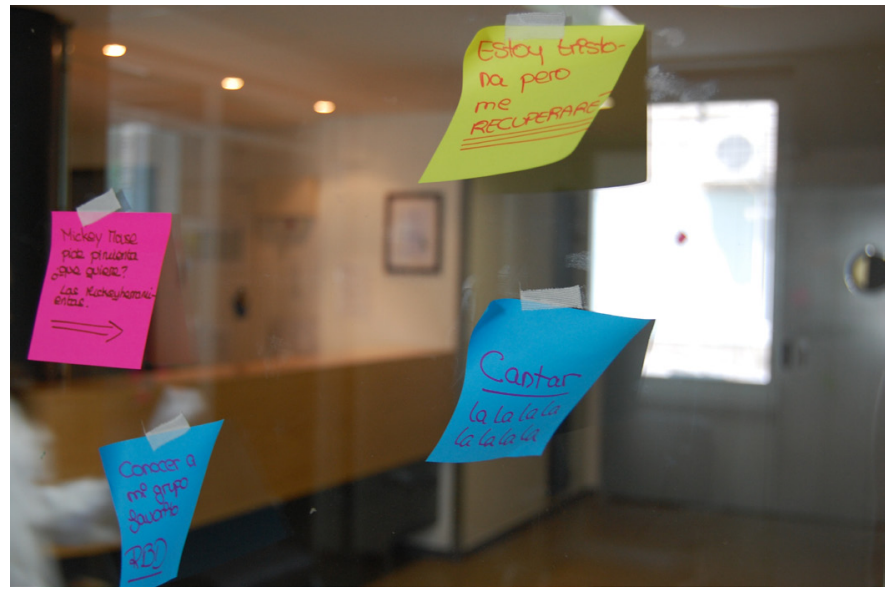

Fig. 5. Imagen del taller sobre "Intervenciones urbanas". 2009, Unidad de Psiquiatría de Adolescentes, Hospital Gregorio Marañón de Madrid. Fondos digitales proyecto curarte $I+D$.

Lo que se presenta a continuación, es una relación de algunos de los talleres diseñados, implementados y evaluados por los educadores e investigadores del proyecto curarte I+D desde el año 2004. Talleres con jóvenes artistas plásticos en hospitales, talleres de estampación, de creación de videojuegos, etc. Propuestas con niños, con adultos, con familiares y con educadores. Con el fin de difundir el trabajo de estos educadores y con la intención de generar comunidades de educadores expertos en el ámbito sanitario, se muestran los siguientes diez talleres:

Proyecto House. Durante los meses de verano, todas las actividades educativas de los niños y adolescentes hospitalizados se interrumpen. En los últimos cinco años, el programa curarte en verano ha diseñado y desarrollado talleres estivales para estos niños y adolescentes. El proyecto House es una propuesta transformadora del espacio hospitalario a través de dinámicas de juego y creación plástica. 
Talleres de Arte con artistas emergentes. El programa de intercambio entre adolescentes hospitalizados en la Unidad de Psiquiatría del hospital Gregorio Marañón de Madrid y jóvenes artistas plásticos, ha iniciado una serie de dinámicas para introducir el Arte Contemporáneo en el espacio hospitalario.

Creación de videojuegos. Pasar del ocio pasivo al ocio activo a través del videojuego es el objetivo de estos talleres que proponen a los niños y adolescentes en los hospitales generar su propio videojuego, sus gráficos, sus personajes y sus propias. historias.

Arte contemporáneo y participación. A través de estos talleres se invitó a los chicos hospitalizados y también a sus familiares a transformar el espacio hospitalario a través de la proyección de imágenes. Estos talleres han derivado en un proyecto con adultos hospitalizados en la Biblioteca de Pacientes del Hospital 12 de Octubre de Madrid.

Camalletes de cine. Talleres con técnicas de animación cinematográficas para acercar la creación audiovisual a niños y adolescentes hospitalizados.

Tunning. En los inicios del proyecto curarte I+D se generaron una serie de materiales de juego creativo especialmente adaptados para su uso hospitalario. A través de los talleres tunning han sido los propios adolescentes hospitalizados los que han participado en la generación de sus propios muñecos.

Talleres de estampación alternativa. Durante los últimos años el proyecto curarte I+D ha desarrollado talleres no tóxicos de grabado y estampación en hospitales y espacios sanitarios. La introducción de estos materiales y técnicas no tóxicas con adolescentes hospitalizados ha puesto de manifiesto las potencialidades creativas de estas técnicas creativas que ahora se están exportando al contexto de la discapacidad y la educación especial.

Talleres de reciclaje con materiales del hospital. Los objetos del hospital, tijeras, bisturíes, mascarillas, tienen connotaciones negativas para los niños hospitalizados. En los talleres plásticos transformamos estos objetos.

Mi ciudad inventada. Experiencia simultánea realizada en talleres de verano en el MUPAI y en la Ciberaula del Hospital Gregorio Marañón. A partir de materiales reciclados (cajas, cartones), los chicos construyeron su propio espacio urbano.Esto no es un juego. Taller para el desarrollo de la creatividad utilizado para la formación de futuros educadores en contextos de salud.

\section{Referencias}

Clift et al. 2009: The state of arts and health in England. Arts \& Health, vol.1, no.1, 6-35

Macnaughton, J, White, M., Stacey, R. 2005: Researching the benefits of arts in health. Health Education, 105 (5), 332-339.

Pascale, P. y Avila, N. 2007: Una experiencia de creatividad con adolescentes hospitalizados: unidad de psiquiatría de adolescentes del Gregorio Marañón. Arte, individuo y sociedad, 19, 207-246.

Ullán, A. y Belver, M.H. 2004: Los niños en los hospitales: espacios, tiempos y juegos en la hospitalización infantil. Madrid: Ediciones Témpora. 